This information is current as of April 26, 2023.

\title{
Incidental Radiologic Findings in the 22q11.2 Deletion Syndrome
}

J.E. Schmitt, J.J. Yi, D.R. Roalf, L.A. Loevner, K. Ruparel, D. Whinna, M.C. Souders, D.M. McDonald-McGinn, E. Yodh, S. Vandekar, E.H. Zackai, R.C. Gur, B.S. Emanuel and R.E. Gur

AJNR Am J Neuroradiol 2014, 35 (11) 2186-2191 doi: https://doi.org/10.3174/ajnr.A4003

http://www.ajnr.org/content/35/11/2186 


\title{
Incidental Radiologic Findings in the 22q11.2 Deletion Syndrome
}

\author{
J.E. Schmitt, J.J. Yi, D.R. Roalf, L.A. Loevner, K. Ruparel, D. Whinna, M.C. Souders, D.M. McDonald-McGinn, E. Yodh, S. Vandekar,
} E.H. Zackai, R.C. Gur, B.S. Emanuel, and R.E. Gur

\begin{abstract}
BACKGROUND AND PURPOSE: The 22q11.2 deletion syndrome is a common genetic microdeletion syndrome that results in cognitive delays and an increased risk of several psychiatric disorders, particularly schizophrenia. The current study investigates the prevalence of incidental neuroradiologic findings within this population and their relationships with psychiatric conditions.
\end{abstract}

MATERIALS AND METHODS: Brain MR imaging from 58 individuals with 22q11.2 deletion syndrome was reviewed by board-certified radiologists by using standard clinical procedures. Intracranial incidental findings were classified into 8 categories and compared with a large typically developing cohort.

RESULTS: The rate of incidental findings was significantly higher $(P<.0001)$ in 22q11.2 deletion syndrome compared with typically developing individuals, driven by a high prevalence of cavum septum pellucidum (19.0\%) and white matter abnormalities (10.3\%). Both of these findings were associated with psychosis in 22q11.2 deletion syndrome.

CONCLUSIONS: Cavum septum pellucidum and white matter hyperintensities are significantly more prevalent in patients with the 22 q11.2 deletion syndrome and may represent biomarkers for psychosis.

ABBREVIATIONS: CSP = cavum septum pellucidum; PNC = Philadelphia Neurodevelopmental Cohort; 22q11DS = 22q11.2 deletion syndrome; TD = typically developing

T he 22q11.2 deletion syndrome (22q11DS, also known as DiGeorge syndrome, velocardiofacial syndrome, and CATCH22 ) is an uncommon genetic disorder occurring in approximately 1:2000-1:4000 live births. ${ }^{1}$ It is typically caused by a sporadic uneven recombination event resulting in hemizygous deletion of approximately 3 megabases on the long arm of chromosome $22 .^{2-4}$ In addition to craniofacial and vascular abnormalities, this deletion of approximately 50 genes results in cognitive delays and

Received February 10, 2014; accepted after revision March 26.

From the Department of Radiology (J.E.S., L.A.L.), Hospital of the University of Pennsylvania, Philadelphia, Pennsylvania; Brain Behavior Laboratory (J.E.S., J.J.Y., D.R.R., K.R., D.W., E.Y., S.V., R.C.G., R.E.G.), Department of Psychiatry, University of Pennsylvania, Philadelphia, Pennsylvania; Department of Psychiatry (J.J.Y.) and Division of Human Genetics (M.C.S., D.M.M.-M., E.H.Z., B.S.E.), Children's Hospital of Philadelphia, Philadelphia, Pennsylvania; Department of Pediatrics (D.M.M.-M., E.H.Z. B.S.E.), University of Pennsylvania School of Medicine, Philadelphia, Pennsylvania; and Department of Pediatrics (D.M.M.-M., E.H.Z., B.S.E.), Perelman School of Medicine, University of Pennsylvania, Philadelphia, Pennsylvania.

This study was supported by National Institutes of Health grants MH087626, MH087636, and T32 and grants MH019112 (J.J.Y.) and EB004311 (J.E.S.).

Please address correspondence to Raquel E. Gur, MD, Brain Behavior Laboratory, 10th Floor, Gates Building, Hospital of the University of Pennsylvania, Philadelphia, PA 19104; e-mail address: raquel@mail.med.upenn.edu

- Indicates open access to non-subscribers at www.ajnr.org

http://dx.doi.org/10.3174/ajnr.A4003 increased risk of several psychiatric diseases, including anxiety, mood disorders, attention deficit, and autistic features. ${ }^{5-8}$ However, perhaps the most striking effect of the $22 \mathrm{q}$ deletion is an approximately 30 -fold increased risk of schizophrenia relative to the general population. , $^{, 10}$

Neuroimaging studies demonstrate consistent anatomic differences between individuals with 22q11DS and typically developing (TD) individuals. Findings include globally decreased cerebral brain volumes, volumetric reductions in the parietal lobe, reduction of cortical thickness in the parietal lobes and orbitofrontal cortex, reduction in the cerebellar vermis hemisphere size, abnormalities in gyral complexity, and white matter hyperintensities. ${ }^{11-16}$ Additionally, prior neuroimaging studies report an increased prevalence of cavum septum pellucidum (CSP) and cavum vergae in 22q11DS, ${ }^{15,17,18}$ an observation also noted in individuals with schizophrenia. ${ }^{19,20}$

Observations such as CSP and white matter hyperintensities are often considered incidental findings, usually of questionable clinical significance. In the TD adult population, the reported rate of incidental findings on neuroanatomic scans is widely variable, ranging from $3 \%$ to $85 \% .{ }^{21-24}$ In a recent prospective investigation of incidental findings, our group estimated the rate of incidental findings to be approximately $10 \%$ in a pediatric and young 
adult TD population. ${ }^{25}$ That study also found an association between psychosis-related symptoms and CSP.

The purpose of the present study was to investigate the rate of incidental findings in a large sample of pediatric and young adult subjects with 22q11DS by using methods similar to those in our prior study in a TD group. To our knowledge, the present study represents the first systematic review of incidental findings in 22q11DS by board-certified neuroradiologists on scans acquired at 3T. Given evidence of CSP as a potential biomarker for schizophrenia and psychosis, we were particularly interested in this finding in subjects with an established genetic predisposition for the disease. Additionally, because of the association between 22q11DS and cardiovascular disease, we also hypothesized that the prevalence of vascular findings would be increased in our sample.

\section{MATERIALS AND METHODS Subjects}

The sample was drawn from a prospective study, Brain-Behavior and Genetic Studies of the 22q11DS, at the University of Pennsylvania and Children's Hospital of Philadelphia. Individuals with the diagnosis of 22q11DS and 8 years of age or older were eligible for the study and were recruited from the 22q and You Center at the Children's Hospital and through social media. Inclusion criteria were the following: 8 years of age or older, English proficiency, stable medical status, and estimated intelligence quotient of $>70$ by the Wide Range Achievement Test IV. ${ }^{26}$ Exclusion criteria were the following: pervasive developmental disorder or intelligence quotient of $<70$ and medical disorders that may affect brain function (eg, uncontrolled seizures, head trauma, CNS tumor, and infection) or visual performance (eg, blindness). Participants with an intelligence quotient of $<70$ were excluded to increase the reliability of the clinical data that focus on neuropsychiatric presentation and neurocognition. Furthermore, to enable comparison with participants without deletions with psychosis spectrum features, we excluded potential participants with significant intellectual disability.

As previously described, ${ }^{27}$ all participants underwent clinical assessment with semistructured interviews. Additionally, participants 12 years of age or older and without conditions interfering with MR imaging (eg, metallic inserts, orthopedic circumstances, poor vision, and pregnancy) underwent neuroimaging. Fiftyeight participants were scanned. Deletion status was confirmed by using multiplex ligation-dependent probe amplification. ${ }^{28}$ Fiftythree patients had the typical 3 megabase and 4 had a 1.5 megabase deletion. For 1 subject, the deletion size could not be determined due to sample quality, though the presence of a deletion was identified by using fluorescence in situ hybridization. All procedures were approved by the institutional review boards of the University of Pennsylvania and the Children's Hospital of Philadelphia. Informed consent/assent was obtained from each participant and accompanying parent for those younger than 18 years at the time of initial evaluation.

\section{Image Acquisition and Analyses}

For each subject, high-resolution axial T1-weighted magnetization-prepared rapid acquisition of gradient echo imaging was ac- quired, with the following parameters: TR/TE, 1810/3.51 ms; TI, $1100 \mathrm{~ms}$; FOV, $180 \times 240 \mathrm{~mm}$; effective resolution, $1 \mathrm{~mm}^{3}$. All subjects were scanned on the same 3T MR imaging scanner (Tim Trio; Siemens, Erlangen, Germany) by using a 32-channel head coil. A board-certified technologist in the Department of Radiology at the Hospital of the University of Pennsylvania performed all scans.

All images were reviewed by board-certified neuroradiologists by using standard clinical procedures, and findings were communicated via standard reports within the medical record. Clinical reports were categorized as the following: 1) normal without incidental findings, 2) incidental findings not requiring follow-up, and 3 ) incidental findings requiring follow-up.

As in prior studies, ${ }^{25}$ incidental findings were categorized as the following: 1) pineal cysts, 2) other cysts (eg, arachnoid cysts), 3) cavum septum pellucidum/cavum vergae, 4) other ventricular abnormalities (ventricular asymmetries or prominence), 5) vascular abnormalities (absent flow voids, aneurysms, developmental venous anomalies, and so forth), and 6) cerebellar abnormalities (eg, hypoplasia, Chiari 1, cerebellar cysts). Two additional categories were added on the basis of prior 22q11DS studies: 7) white matter abnormalities, and 8) subcortical abnormalities. Extracranial findings were not included in the analyses.

\section{Statistical Analysis}

All analyses were performed in the $\mathrm{R}$ statistical environment (http://www.r-project.org/). ${ }^{29}$ Prevalence rates were calculated for individual incidental finding categories and for the aggregate prevalence of all incidental findings within the 22q11DS population. Demographic differences were tested by using ANOVA for age and the Fisher exact test for proportions (sex, race). An $\alpha$ of .05 was set as the threshold for statistical significance. Additionally, logistic regression was performed to test whether demographic variables or their interactions predicted incidental findings (generalized linear model function in R). Linear and nonlinear effects of age, sex, race, and interaction terms were included in the full model. All models were evaluated for goodness of fit by using the Hosmer-Lemeshow test. ${ }^{30}$ Best fit submodels were determined via combined stepwise regression by using Akaike information criteria as the test statistic. ${ }^{31}$

The 22q11DS dataset was then compared with data on incidental findings from the Philadelphia Neurodevelopmental Cohort (PNC), a TD sample of children and young adults 8-21 years of age comprising 1445 individuals. Details on this sample are described elsewhere. ${ }^{32}$ Logistic regression models were performed to test whether group (PNC versus 22q11DS) predicted the presence of incidental findings. These models included linear and nonlinear effects of age, sex, and race to control for these potential confounds. Because our original report on the PNC did not explicitly include categories for WM and subcortical abnormalities, the original PNC reports were re-reviewed to identify any potential under-reported findings in these categories.

Finally, we tested the associations of incidental findings and the prevalence of psychiatric disease. On the basis of our a priori hypothesis that CSP and psychosis were related, we tested the hypothesis that CSP is associated with psychotic features. Specifically, logistic regression was used to predict whether subjects who 
Table 1: Demographic differences in 22q11DS with and without incidental findings

\begin{tabular}{lccc}
\hline & No Incidentals & Incidentals & $\boldsymbol{P}$ Value \\
\hline No. & $31(53 \%)$ & $27(47 \%)$ & \\
Age (mean years \pm SD) & $22.8(8.1)$ & $22.4(7.1)$ & .8512 \\
Sex & & & .5990 \\
$\quad$ Male & $17(55 \%)$ & $12(44 \%)$ & \\
$\quad$ Female & $14(45 \%)$ & $15(56 \%)$ & \\
Race & & & .9999 \\
$\quad$ White & $27(87 \%)$ & $19(88 \%)$ & \\
$\quad$ African American & $3(10 \%)$ & $2(7 \%)$ & \\
$\quad$ Other & $1(3 \%)$ & $1(4 \%)$ & \\
\hline
\end{tabular}

Table 2: Frequency of incidental findings in 21q11DS

\begin{tabular}{lcc}
\hline \multicolumn{1}{c}{ Finding } & Count & Prevalence \\
\hline Pineal cyst & 2 & $2.58 \%$ \\
Other cysts & 0 & $0 \%$ \\
Cavum septum pellucidum & 11 & $19.0 \%$ \\
Ventricular abnormalities & 5 & $8.62 \%$ \\
Other CSF abnormalities & 1 & $1.72 \%$ \\
Vascular abnormalities & 5 & $8.62 \%$ \\
Cerebellar abnormalities & 0 & $0 \%$ \\
White matter abnormalities & 6 & $10.34 \%$ \\
Subcortical & 2 & $3.45 \%$ \\
All subjects with incidental findings & 27 & $46.6 \%$ \\
\hline
\end{tabular}

carry psychiatric diagnoses with severe psychotic features (schizophrenia, schizoaffective disorder, or major depression with psychotic features) have increased rates of CSP. This analysis was repeated for $\mathrm{WM}$ abnormalities. We then tested the predictive ability of incidental findings for categories of other psychiatric diagnoses (attention deficit/hyperactivity disorder, anxiety disorders, prodromal psychosis, and severe psychosis) via multivariate generalized linear models in $\mathrm{R}$.

\section{RESULTS}

Of the 58 subjects with 22q11DS imaged, 27 had incidental findings $(47 \%)$. There were no statistically significant differences in age, sex, or race between subjects with and without incidentals (Table 1). CSP and WM abnormalities were the most common incidental findings (Table 2). Secondary review of the 11 individuals with CSP demonstrated that CSP et vergae was present in all reported cases (Fig 1). Representative examples of other common incidental findings are provided in Fig 2. Five individuals in the sample presented with multiple incidental findings: 1) CSP and ventricular prominence, 2) white matter hyperintensities and a 12-mm pineal cyst, 3) multiple white matter hyperintensities and a nonspecific hypointensity in the left thalamus, 4) CSP and absent left ICA flow, and 5) ventricular asymmetry and a nonspecific white matter hyperintensity.

Compared with the TD population, the overall rate of incidental findings was $>4$ times greater in the 22q11DS population $(P<$ $.0001)$. Similar to prior studies, these differences were largely driven by an increased rate of CSP and white matter abnormalities (Table 3). Ventricular abnormalities also were more common in the 22q11DS population. In our sample, differences in infratentorial abnormalities were not statistically significant; no clinical abnormalities were reported in the cerebellum in our 22q11DS sample.

Figure 3 summarizes the relative prevalence of incidental find-
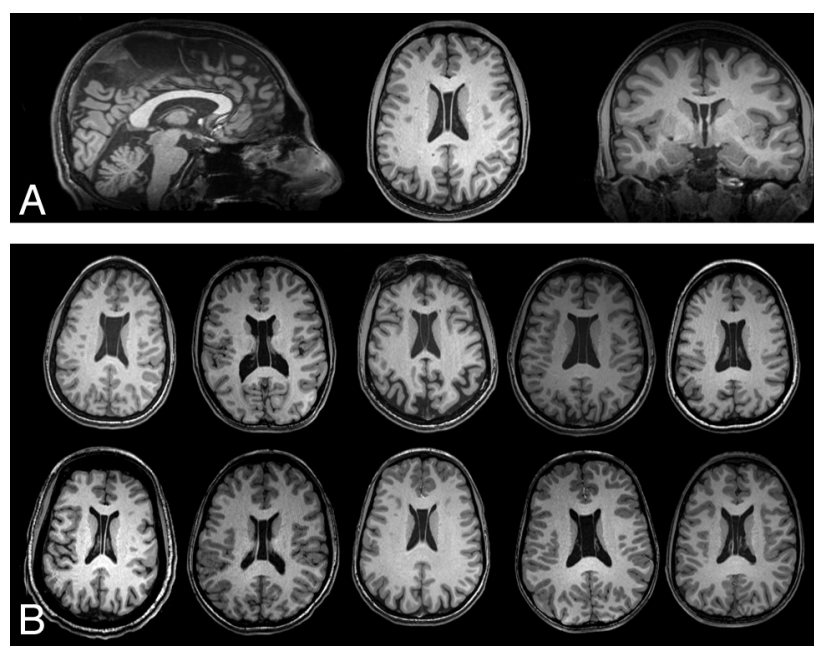

FIG 1. High prevalence of cavum septum pellucidum et vergae in 22q11DS. A, Triplanar view centered on the lateral ventricles in a subject with CSP et vergae. $B$, Single representative axial sections from 10 other subjects with this finding.
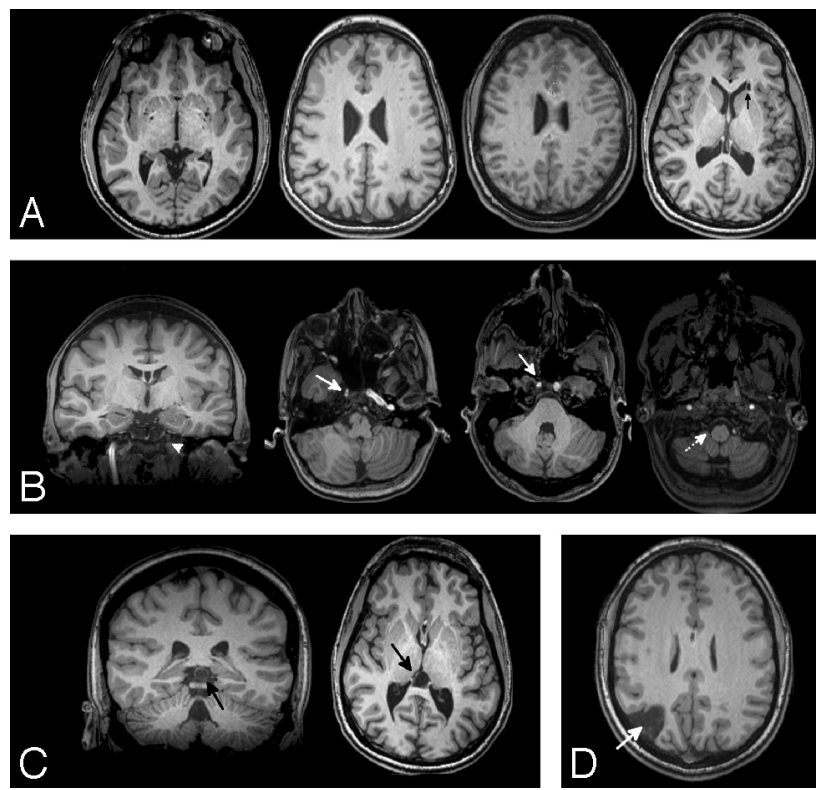

FIG 2. Incidental findings in 11 representative individuals with 22q11DS Other than CSP, white matter abnormalities $(A)$ were the most common finding. Scattered WM hypointensities were most frequent, with 1 subject having a nonspecific cyst in the periventricular WM (arrow). Vascular abnormalities $(B)$ were seen in $8.6 \%$ of the population and included an absent left ICA flow void (arrowhead) with a CSP also noted, asymmetries in the ICA (solid arrows), and absence of a right vertebral flow void (dotted arrow). Pineal cysts $(C)$ were also seen. A single subject had a known right posterior infarct $(D)$.

ings in 22q11DS by psychiatric diagnosis. Of the 11 subjects with CSP (19\%), 3 carried a diagnosis of schizophrenia, psychotic depression, or schizoaffective disorder $(27 \%)$. In contrast, these diagnoses were seen in only 3 of the remaining 53 subjects (6\%); this finding was statistically significant $(P=.04)$. Similarly, of the 6 individuals with incidental white matter abnormalities, 3 had diagnoses with psychotic features (50\%), significantly greater than those without these abnormalities $(6 \%, P$ value $=.01)$. Notably, there was no comorbidity between CSP and WM abnormalities in our sample, but all subjects with psychosis had either CSP or WM 
abnormalities. There were no statistically significant associations between other psychiatric diagnoses and incidental findings as determined by post hoc multivariate logistic regression.

\section{DISCUSSION}

To our knowledge, the current study represents the first neuroradiologic evaluation of incidental findings at a high field strength in 22q11DS. We found a higher rate of incidental findings within this population relative to a control population when analyzed by using similar methods. The increased rate of incidental findings was driven by a higher prevalence of cavum septum pellucidum, white matter hyperintensities, and vascular abnormalities, findings that have been noted in prior samples with 22q11DS. We also observed an increased rate of psychiatric diagnoses with psychotic features in subjects with 22q11DS with either CSP or WM abnormalities.

Cavum septum pellucida were first described in 1671 by Franciscus Sylvius. ${ }^{33}$ In prenatal life, a CSP is a normal anatomic structure. Identification of a CSP is considered part of a standard evaluation during fetal sonography, and its absence is associated with several abnormalities including septo-optic dysplasia, abnormalities of the corpus callosum, and hydrocephalus. ${ }^{34}$ During typical development, the CSP slowly closes following birth; the septal leaflets fuse in approximately $15 \%$ of the population within 1 month following birth and in $85 \%$ by 6 months. ${ }^{35}$ The reported prevalence of a persistent CSP in late childhood and adulthood is

Table 3: Comparison in incidental findings between subjects with 22q11DS and typically developing (PNC) sample

\begin{tabular}{lccc}
\hline & 22q11DS & PNC & P Value \\
\hline Pineal cyst & $2.58 \%$ & $2.43 \%$ & .6213 \\
Other cyst & $0 \%$ & $1.36 \%$ & .9855 \\
Cavum septum pellucidum & $18.96 \%$ & $1.14 \%$ & $<.0001$ \\
Vascular abnormalities & $8.62 \%$ & $2.57 \%$ & .0173 \\
White matter & $11.53 \%$ & $0.56 \%$ & $<.0001$ \\
Subcortical & $3.45 \%$ & $0.06 \%$ & .0960 \\
Cerebellar & $0 \%$ & $2.36 \%$ & .9860 \\
All incidental & $46.55 \%$ & $10.72 \%{ }^{\mathrm{a}}$ & $<.0001$ \\
\hline
\end{tabular}

Note:-VCFS indicates velocardiofacial syndrome.

a Previously reported as $10.57 \%$, with WM and subcortical abnormalities included in the current analyses.

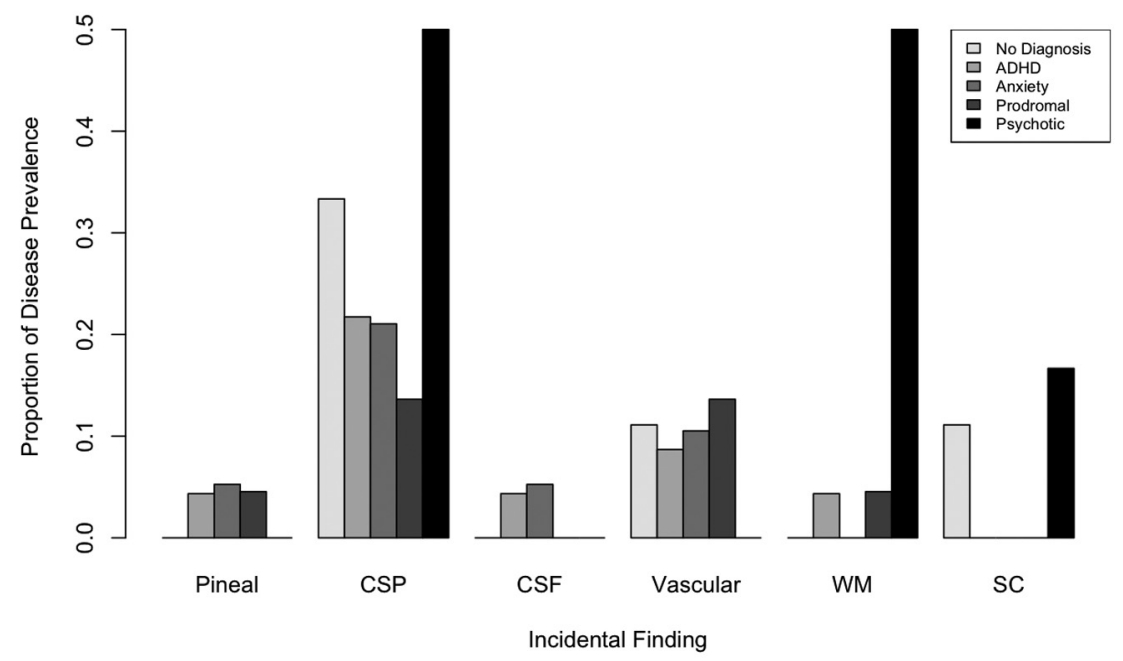

FIG 3. Proportion of incidental findings in 22q11DS split by psychiatric diagnosis. highly variable, ranging from $2 \%$ to nearly $60 \% .^{36,37}$ The high variability in reported prevalence is at least in part due to disagreement on whether persistent CSP represents a true radiographic abnormality. For example, many of the largest studies on incidental neuroimaging findings do not mention the CSP at all ${ }^{23,24}$ or explicitly exclude the CSP from analysis. ${ }^{22}$

The precise developmental trigger that results in fusion of the cavum remains uncertain. It is hypothesized that growth of midline structures such as the corpus callosum, fornix, and hippocampus result in increased pressure on the leaflets of the septum, ultimately resulting in fusion. ${ }^{38,39}$ Thus, a persistent CSP may be a proxy for aberrant development of midline structures or possibly for abnormal global cerebral growth. CSP has been associated with several disorders including schizophrenia, Sotos syndrome, Apert syndrome, fetal alcohol syndrome, and chronic head trauma. ${ }^{40-42}$ In a recent meta-analysis of the extant literature on schizophrenia and CSP, Trzesniak et $\mathrm{al}^{20}$ found that a large CSP was associated with an increased risk of schizophrenia spectrum disorders. Schizophrenia has also been associated with decreased size of several midline structures such as the hippocampus and corpus callosum, again suggesting that CSP may be related to abnormalities in midline development in this condition. ${ }^{20}$

In the 22q11DS population specifically, several prior studies noted an increased incidence of CSP. ${ }^{15,18,38,43}$ For example, van Amelsvoort et $\mathrm{al}^{15}$ estimated a $40 \%$ prevalence of CSP in a sample of 10 subjects with 22q11DS, compared with 9\% in TD controls, though this finding did not reach statistical significance. In a multisite study of 45 children with 22q11DS, a prevalence of $84 \%$ was reported. ${ }^{18}$ Beaton et al ${ }^{18}$ measured CSP volume and length differences between 45 individuals with 22q11DS and matched TD controls, finding quantitative increases in both measures within the 22q11DS population. There also is some evidence that CSP and psychosis are associated in patients with 22q11DS: Chow et al ${ }^{17}$ noted CSP in 5/11 subjects with either a diagnosis of schizophrenia or schizoaffective disorder, though the lack of a control group prevented tests of statistical significance. Our data provide statistical evidence that there is an association between CSP and psychosis within this population.

We also observed increased rates of vascular abnormalities in 22q11DS, predominantly due to absent intracranial flow voids. Extracranial cardiovascular abnormalities are common in this condition, with $70 \%$ of individuals having congenital heart disease. ${ }^{1}$ Abnormal placement of the extracranial internal carotid arteries $^{44}$ and kinked or tortuous vertebral arteries ${ }^{45}$ have also been reported, occasionally complicating the pharyngeal surgeries that are common in this condition. The available data are more limited within the calvaria, but several abnormalities in intracranial vascularity have been reported, including hypoplasia of the circle of Willis and other major intracranial vessels. ${ }^{46}$ These vascular abnormalities 
may be in part responsible for downstream neuroanatomic abnormalities that have been previously described in 22q11DS such as volume loss and white matter hyperintensities. ${ }^{47}$ Further research into this potential link between intracranial vascular anomalies and brain abnormalities, such as with noninvasive MR angiography and perfusion MR imaging, may be of value.

Additionally, there was an increased incidence of WM abnormalities in 22q11DS. Several prior studies have noted this finding in smaller samples or case reports. ${ }^{15,48,49}$ For example, van Amelsvoort et $\mathrm{al}^{15}$ noted a rate of hyperintensities at approximately twice that in their control population, though the finding did not reach statistical significance. Chow et $\mathrm{al}^{17}$ observed WM hyperintensities in 9/10 subjects with schizophrenia or schizoaffective disorder. The pathophysiology of white matter hyperintensities is incompletely understood at present, but pathologic correlation in the general population has suggested that they occur in regions of gliosis, axonal loss, and demyelination, likely secondary to perivascular damage. ${ }^{50}$ In the TD population, the prevalence of focal white matter abnormalities increases with age, and increased WM burden is associated with an increased risk of dementia, cerebrovascular disease, mood disorders, and death. ${ }^{51,52}$ Because subjects with 22q11DS have an increased risk of vascular abnormalities, it is tempting to conclude that focal white matter abnormalities in this population may also represent premature perivascular damage due to a genetic predisposition. However, this hypothesis has not been tested directly because there are no large studies providing radiologic-pathologic correlation within the 22q11DS population specifically.

Although the current study supports several prior findings in the literature in a relatively large sample, there are some limitations. First, our estimates of a few incidental findings are lower than those reported elsewhere. These differences may be related to subject ascertainment procedures. Specifically, our study selected for higher functioning subjects and therefore may have underestimated the true prevalence of any incidentals with direct impact on brain function. The lack of cerebellar findings, in particular, was unexpected, given prior reports of vermian and cerebellar hemispheric abnormalities. ${ }^{13,16,53}$ Second, our comparisons between the 22q11DS and PNC samples controlled for demographic variables by using statistical methods rather than by directly matching for age, sex, and race. The higher mean age of the 22q11DS group (22.6 versus 14.8 years) could potentially contribute to the increased rate of white matter abnormalities in this group, though the natural history of these findings within the TD population makes this less likely because they generally develop after 40 years of age. ${ }^{52}$ In contrast, incomplete control of group age differences would be expected to underestimate the relative rate of CSP in the 22q11DS on the basis of its natural history of decreasing size with age. ${ }^{35}$

\section{CONCLUSIONS}

The current study confirms an increased rate of incidental brain MR imaging findings within the 22q11DS population in a large sample. In concordance with prior literature, cavum septum pellucidum and white matter hyperintensities drive the increases in the prevalence of incidental findings. Both cavum septum pellucidum and white matter abnormalities are found with increased prevalence in 22q11DS with psychosis and may represent potential biomarkers for schizophrenia and psychosis in 22q11DS. Future longitudinal studies within this sample could help further refine our understanding of these putative risk factors.

Disclosures: James E. Schmitt—RELATED: T32 Training Grant from the National Institutes of Health (EB004311, to Mitch Schnall), ${ }^{*}$ UNRELATED: a Fellow Grant submission to the Radiological Society of North America (Imaging Genomic Analysis of the 22qliDS Syndrome). James Yi-RELATED: National Institute of Mental Health grant (T32 MH019112).* David Roalf_RELATED: a postdoctoral fellow during part of this work, supported by National Institute of Mental Health grant T32 MH019112 (Principal Investigator: Raquel E. Gur). * Margaret C. Souders—RELATED: National Institutes of Health grant support.* Ruben C. Gur-RELATED: grant support from the National Institute of Mental Health,* UNRELATED: medicolegal services for death penalty defense. Beverly S. Emanuel-These studies were supported in part by an National Institutes of Health/National Institute of Mental Health U01 Grant MH 87636.* Raquel E. Gur-RELATED and UNRELATED: grants from the National Institutes of Health. * *oney paid to the Institution.

\section{REFERENCES}

1. Shprintzen RJ. Velo-cardio-facial syndrome: $\mathbf{3 0}$ years of study. Dev Disabil Res Rev 2008;14:3-10

2. Driscoll D, Salvin J, Sellinger B, et al. Prevalence of 22q11 microdeletions in DiGeorge and velocardiofacial syndromes: implications for genetic counselling and prenatal diagnosis. J Med Genet 1993;30:813-17

3. Edelmann L, Pandita RK, Morrow BE. Low-copy repeats mediate the common 3-Mb deletion in patients with velo-cardio-facial syndrome. Am J Hum Genet 1999;64:1076-86

4. Shaikh TH, Kurahashi H, Saitta SC, et al. Chromosome 22-specific low copy repeats and the 22q11.2 deletion syndrome: genomic organization and deletion endpoint analysis. Hum Mol Genet 2000;9:489-501

5. Eisenberg DP, Jabbi M, Berman KF. Bridging the gene-behavior divide through neuroimaging deletion syndromes: velocardiofacial (22q11.2 deletion) and Williams (7q11.23 deletion) syndromes. Neuroimage 2010;53:857-69

6. Gothelf D, Furfaro JA, Penniman LC, et al. The contribution of novel brain imaging techniques to understanding the neurobiology of mental retardation and developmental disabilities. Ment Retard Dev Disabil Res Rev 2005;11:331-39

7. Gothelf D, Lombroso PJ. Genetics of childhood disorders. XXV. Velocardiofacial syndrome. J Am Acad Child Adolesc Psychiatry 2001;40:489-91

8. Baker KD, Skuse DH. Adolescents and young adults with 22q11 deletion syndrome: psychopathology in an at-risk group. Br J Psychiatry 2005;18:115-20

9. Murphy KC, Jones LA, Owen MJ. High rates of schizophrenia in adults with velo-cardio-facial syndrome. Arch Gen Psychiatry 1999;56:940-45

10. Bassett A, Chow E. 22Q11 deletion syndrome: a genetic subtype of schizophrenia. Biol Psychiatry 1999;46:882-91

11. Gothelf D, Schaer M, Eliez S. Genes, brain development and psychiatric phenotypes in velo-cardio-facial syndrome. Dev Disabil Res Rev 2008;14:59-68

12. Schaer M, Schmitt JE, Glaser B, et al. Abnormal patterns of cortical gyrification in velo-cardio-facial syndrome (deletion 22q11.2): an MRI study. Psychiatry Res 2006;146:1-11

13. Eliez S, Schmitt JE, White CD, et al. Children and adolescents with velocardiofacial syndrome: a volumetric MRI study. Am J Psychiatry 2000;157:409-15

14. Bearden CE, van Erp TG, Dutton RA, et al. Mapping cortical thickness in children with 22q11.2 deletions. Cerebral Cortex 2007:17:1889-98

15. van Amelsvoort T, Daly E, Robertson D, et al. Structural brain abnormalities associated with deletion at chromosome 22q11: quan- 
titative neuroimaging study of adults with velo-cardio-facial syndrome. Br J Psychiatry 2001;178:412-19

16. Eliez S, Schmitt JE, White CD, et al. A quantitative MRI study of posterior fossa development in velocardiofacial syndrome. Biol Psychiatry 2001;49:540-46

17. Chow EW, Mikulis DJ, Zipursky RB, et al. Qualitative MRI findings in adults with 22q11 deletion syndrome and schizophrenia. Biol Psychiatry 1999;46:1436-42

18. Beaton EA, Qin Y, Nguyen V, et al. Increased incidence and size of cavum septum pellucidum in children with chromosome 22q11.2 deletion syndrome. Psychiatry Res 2010;181:108-13

19. Galarza M, Merlo AB, Ingratta A, et al. Cavum septum pellucidum and its increased prevalence in schizophrenia: a neuroembryological classification. J Neuropsychiatry Clin Neurosci 2004;16:41-46

20. Trzesniak C, Oliveira IR, Kempton MJ, et al. Are cavum septum pellucidum abnormalities more common in schizophrenia spectrum disorders? A systematic review and meta-analysis. Schizophr Res 2011;125:1-12

21. Seki A, Uchiyama H, Fukushi T, et al. Incidental findings of brain magnetic resonance imaging study in a pediatric cohort in Japan and recommendation for a model management protocol. J Epidemiol 2010:20(supplement 2):S498-504

22. Morris Z, Whiteley W, Longstreth WT Jr, et al. Incidental findings on brain magnetic resonance imaging: systematic review and metaanalysis. BMJ 2009;33:b3016

23. Vernooij MW, Ikram MA, Tanghe HL, et al. Incidental findings on brain MRI in the general population. $N$ Engl J Med 2007;357: 1821-28

24. Katzman GL, Dagher AP, Patronas NJ. Incidental findings on brain from 1000 asymptomatic volunteers. JAMA 1999;282:36-39

25. Gur R, Kaltman D, Melhem E, et al. Incidental findings in youths volunteering for brain MRI research. AJNR Am J Neuroradiol 2013;34:2021-25

26. Wilkinson G, Robertson G. Wide Range Achievement Test. 4th ed. Lutz: Psychological Assessment Resources; 2006

27. Tang SX, Yi JJ, Calkins ME, et al. Psychiatric disorders in 22q11.2 deletion syndrome are prevalent but undertreated. Psychol Med 2013:1-11

28. Jalali GR, Vorstman JA, Errami A, et al. Detailed analysis of 22q11.2 with a high density MLPA probe set. Human Mutat 2008;29:433-40

29. R Core Team. R: A Language and Environment for Statistical Computing. Vienna, Austria: R Foundation for Statistical Computing; 2014. http://www.R-project.org. Accessed January 24, 2014

30. Lemeshow S, Hosmer D. A review of goodness of fit statistics for use in the development of logistic regression models. Am J Epidemiol 1982;115:92-106

31. Akaike H. Factor analysis and AIC. Psychometrika 1987;52:317-32

32. Satterthwaite TD, Elliott MA, Ruparel K, et al. Neuroimaging of the Philadelphia neurodevelopmental cohort. Neuroimage 2014;86: $544-53$

33. Sylvius F. Opera Medica, Tam Hactenus Inedita, Quam Variis Locis et Formis Edita. Amsterdam: Aput Danielem Elsevirium et Abrahamum Wolfgang; 1679

34. Winter TC, Kennedy AM, Byrne J, et al. The cavum septi pellucidi: why is it important? J Ultrasound Med 2010;29:427-44
35. Saba L, Anzidei M, Raz E, et al. MR and CT of brain's cava. J Neuroimaging 2013;23:326-35

36. Aldur MM, Gürcan F, Başar R, et al. Frequency of septum pellucidum anomalies in non-psychotic population: a magnetic resonance imaging study. Surg Radiol Anat 1999;21:119-23

37. Born CM, Meisenzahl EM, Frodl T, et al. The septum pellucidum and its variants: an MRI study. Eur Arch Psychiatry Clin Neurosci 2004;254:295-302

38. Shashi V, Muddasani S, Santos CC, et al. Abnormalities of the corpus callosum in nonpsychotic children with chromosome 22q11 deletion syndrome. Neuroimage 2004;21:1399-406

39. Bodensteiner JB, Schaefer GB. Wide cavum septum pellucidum: marker of disturbed brain development. Pediatr Neurol 1990;6: 391-94

40. May FS, Chen QC, Gilbertson MW, et al. Cavum septum pellucidum in monozygotic twins discordant for combat exposure: relationship to posttraumatic stress disorder. Biol Psychiatry 2004;55: $656-58$

41. Schaefer GB, Bodensteiner JB, Buehler B a, et al. The neuroimaging findings in Sotos syndrome. Am J Med Genet 1997:68:462-65

42. Johnson VP, Swayze VW II, Sato Y, et al. Fetal alcohol syndrome craniofacial and central nervous system manifestations. Am J Med Genet 1996;61:329-39

43. Campbell LE, Daly E, Toal F, et al. Brain and behaviour in children with 22q11.2 deletion syndrome: a volumetric and voxel-based morphometry MRI study. Brain 2006:129(pt 5):1218-28

44. MacKenzie-Stepner K, Witzel M, Stringer D, et al. Abnormal carotid arteries in the velocardiofacial syndrome: a report of three cases. Plas Reconstr Surg 1987;80:347-51

45. Mitnick R, Bello J, Golding-Kushner KJ, et al. The use of magnetic resonance angiography prior to pharyngeal flap surgery in patients with velocardiofacial syndrome. Plas Reconstr Surg 1996;97:908-19

46. Shprintzen RJ. Velo-cardio-facial syndrome: a distinctive behavioral phenotype. Ment Retard Dev Disabil Res Rev 2000;6:142-47

47. Tan GM, Arnone D, McIntosh AM, et al. Meta-analysis of magnetic resonance imaging studies in chromosome 22q11.2 deletion syndrome (velocardiofacial syndrome). Schizophr Res 2009:115:173-81

48. Altman DH, Altman NR, Mitnick RJ, et al. Further delineation of brain anomalies in velo-cardio-facial syndrome. Am J Med Genet 1995;60:174-75

49. Kates WR, Burnette CP, Jabs EW, et al. Regional cortical white matter reductions in velocardiofacial syndrome: a volumetric MRI analysis. Biol Psychiatry 2001;49:677-84

50. Fazekas F, Kleinert R, Offenbacher H, et al. Pathologic correlates of incidental MRI white matter signal hyperintensities. Neurology 1993;43:1683-89

51. Dupont RM, Butters N, Schafer K, et al. Diagnostic specificity of focal white matter abnormalities in bipolar and unipolar mood disorder. Bio Psychiatry 1995;38:482-86

52. Debette S, Markus H. The clinical importance of white matter hyperintensities on brain magnetic resonance imaging: systematic review and meta-analysis. BMJ 2010:341

53. Mitnick RJ, Bello JA, Shprintzen RJ. Brain anomalies in velo-cardiofacial syndrome. Am J Med Genet 1994;54:100-06 